Supporting information for:

\title{
Self-Diffusion of Associating Star-Shaped Polymers
}

Shengchang Tang, ${ }^{\dagger}$ Axel Habicht, ${ }^{\S}$ Shuaili Li, ${ }^{\dagger}$ Sebastian Seiffert, ${ }^{\S *}$ and Bradley D. Olsen ${ }^{\dagger *}$

${ }^{\dagger}$ Department of Chemical Engineering, Massachusetts Institute of Technology, 77

Massachusetts Avenue, Cambridge, Massachusetts 02139, USA.

$\S$ Institute of Physical Chemistry, Johannes Gutenberg-Universität Mainz, Duesbergweg 10-14,

D-55128 Mainz, Germany.

To whom correspondence should be addressed: B.D.O (bdolsen@mit.edu) and S.S (sebastian.seiffert@uni-mainz.de). 
Table of Contents

A. Supporting Figures Cited in the Main Text 2

B. Additional Experimental Details 6

C. Additional Calculation Details 9

D. References 11 


\section{A. Supporting Figures}
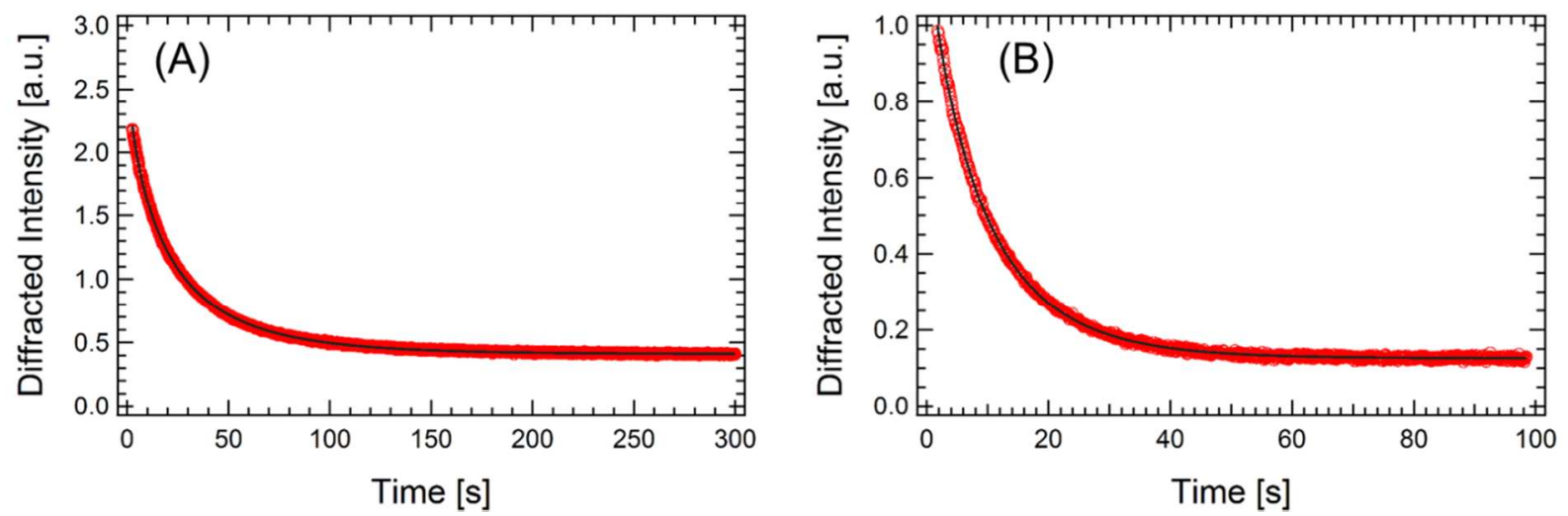

Figure S1. Representative decays of the diffracted scattering intensity over time as probed by

FRS for the diffusion of associating star-shaped PEG polymers in model transient networks. The red open circles are experimental data, and the black lines are fits to a stretched exponential function. (A) Self-diffusion, gel concentration $15 \%(\mathrm{w} / \mathrm{v})$, measurement at a beam angle of 6.88 degrees. (B) Tracer diffusion, gel concentration $20 \%(\mathrm{w} / \mathrm{v})$, measurement at a beam angle of 6.88 degrees. Both measurements were performed at $25^{\circ} \mathrm{C}$.
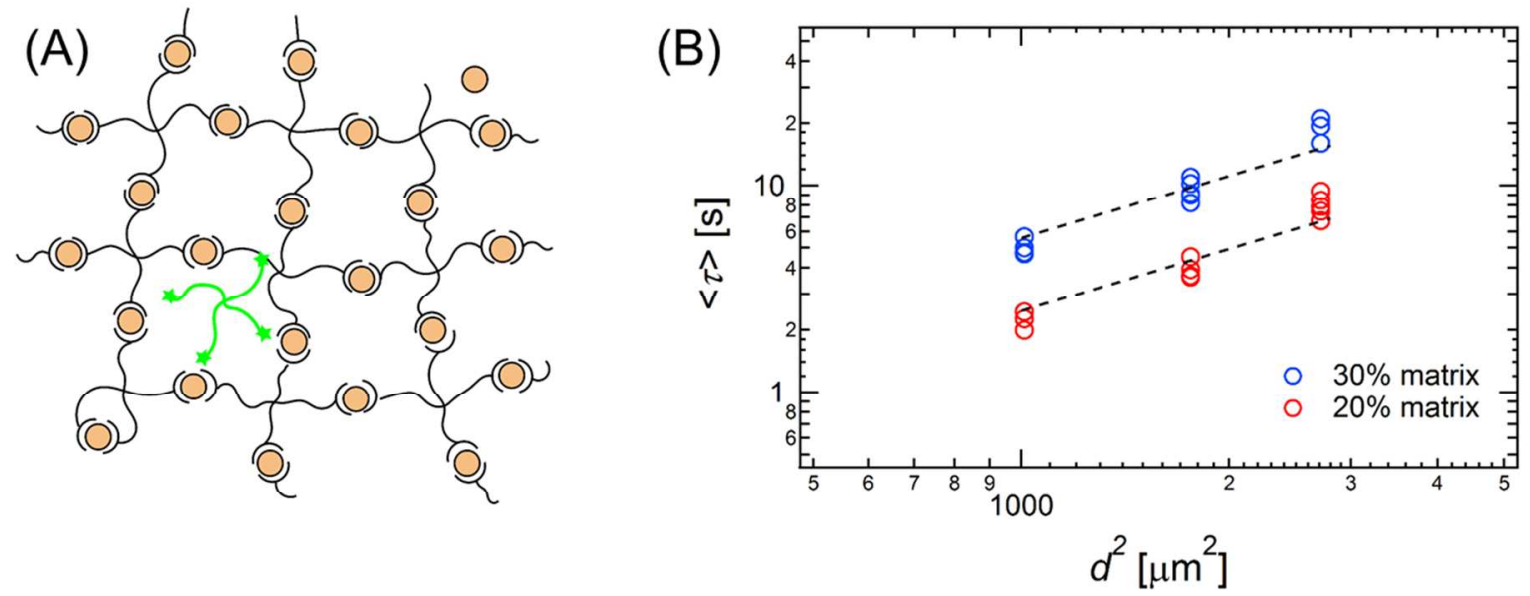

Figure S2. (A) Schematic presentation of an unassociating four-arm PEG polymer (labeled in green) diffusing in a model transient network formed by terpyridine-capped four-arm PEG 
complexed with $\mathrm{Zn}^{2+}$ in DMF. (B) Corresponding $<\tau>$ vs. $d^{2}$ relation. The matrix concentrations are 20 and $30 \%(\mathrm{w} / \mathrm{v})$, respectively. Dashed lines are linear regressions of slope 1 that determine the tracer diffusion coefficients. While only Fickian diffusion is observed within a narrow window of length and time scales accessible by FRS experiments, anomalous diffusion should not be expected since there is a lack of associative interactions between the labeled, nonsticky polymers and the surrounding matrix. Diffusion at lower concentrations, 10 and $15 \%$ $(\mathrm{w} / \mathrm{v})$, is too fast to be accurately measured.

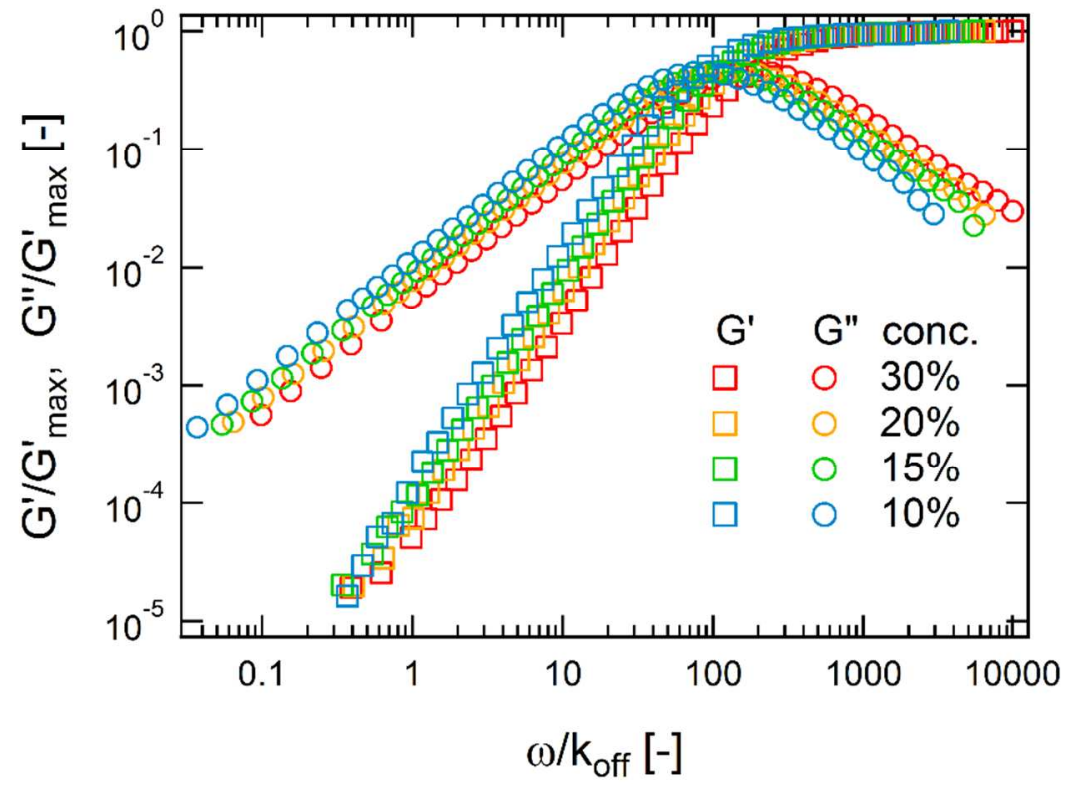

Figure S3. Rheology data plotted in a reduced parameter space. As seen in the figure, however, renormalization by the molecular dissociation rate $k_{\text {off }}$ does not yield perfect collapse of the data. The reference temperature is $25^{\circ} \mathrm{C}$. 


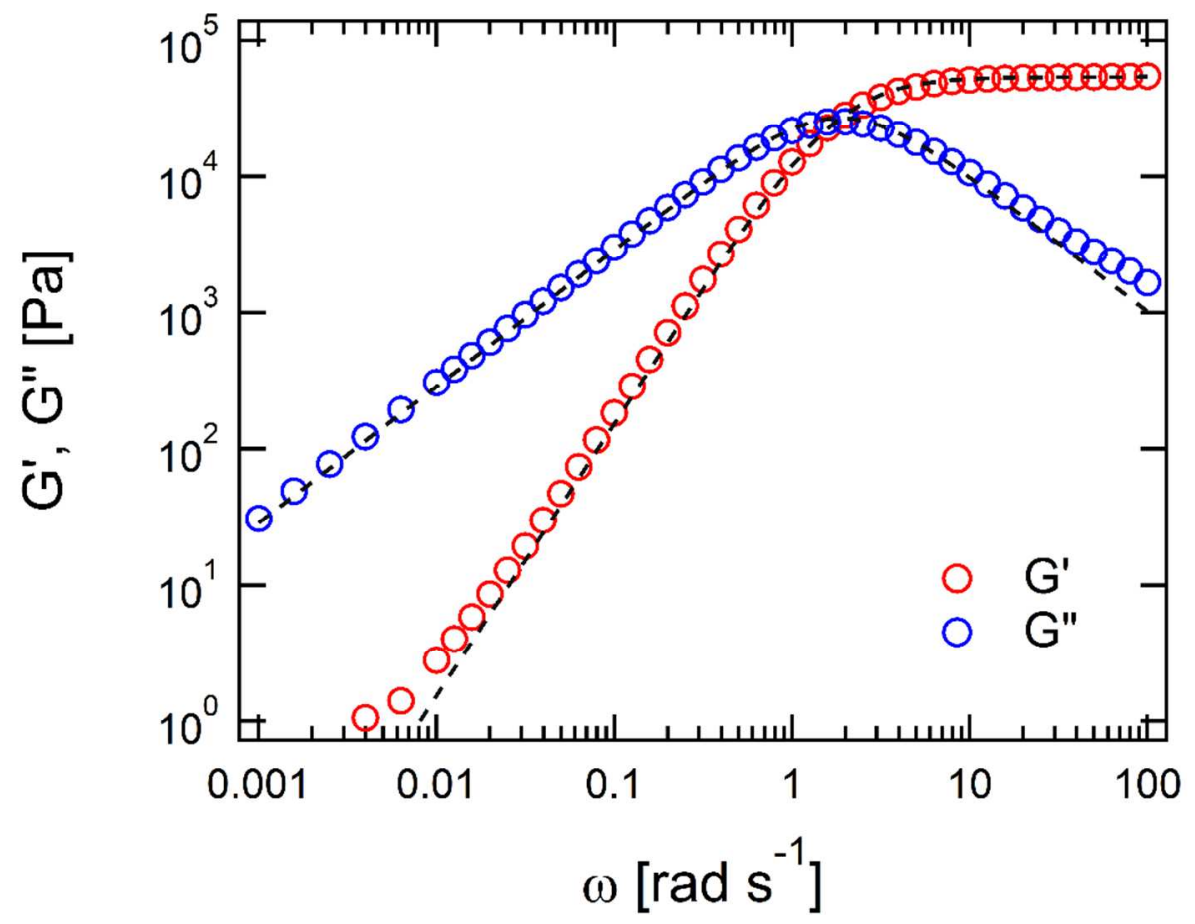

Figure S4. Fitting the single Maxwell model to the dynamic moduli of gels at $30 \%(w / v)$ at $25^{\circ} \mathrm{C}$. There are noticeable differences between the fit (black dashed line) and the experimental data (open circles) 


\section{B. Additional Experimental Details}

\section{B-1. Synthesis of four-arm PEG polymers with all chain ends capped by nitrobenzofurazan}

(NBD)

Commercial tetra-arm PEG-OH (Creative PEGWorks, $M_{\mathrm{n}}=10 \mathrm{~kg} \mathrm{~mol}^{-1}, M_{\mathrm{w}} / M_{\mathrm{n}}=1.03$, $4.57 \mathrm{~g}, 0.46 \mathrm{mmol}$ ) was end-labeled with NBD in two steps (Scheme S1). In the first step, the polymer was dissolved in dry dichloromethane $(80 \mathrm{~mL})$, dry pyridine $(0.48 \mathrm{~mL}, 5.4 \mathrm{mmol})$ was added to the solution, followed by addition of $p$-nitrophenyl chloroformate $(0.58 \mathrm{~g}, 2.8 \mathrm{mmol})$. The resulting suspension was stirred at room temperature overnight, the precipitate was filtered off, and brine $(250 \mathrm{~mL})$ was added to the filtrate. The mixture was extracted with dichloromethane $(2 \times 400 \mathrm{~mL})$, the organic layer was dried over $\mathrm{MgSO}_{4}$ and then concentrated in vacuo. The product, tetra-arm PEG- $p$-nitrophenylcarbonate, was precipitated by pouring the concentrate into cold diethyl ether, yielding a slightly violet solid 1 (4.14 g, 86\%). ${ }^{1} \mathrm{H}$ NMR (700 MHz, DMSO-d $\left.d_{6} \delta\right): 8.32(\mathrm{~d}, J=9.1 \mathrm{~Hz}, 8 \mathrm{H}), 7.57(\mathrm{~d}, J=9.1 \mathrm{~Hz}, 8 \mathrm{H}), 3.31(\mathrm{~m}, 902 \mathrm{H}) \mathrm{ppm}$; $\mathrm{IR}$ $\left(\right.$ ATR, $\left.\mathrm{cm}^{-1}\right): v=2880(\mathrm{~m}), 2360(\mathrm{w}), 2342(\mathrm{w}), 1965(\mathrm{w}), 1769(\mathrm{w}), 1524(\mathrm{w}), 1466(\mathrm{~m}), 1359$ (w), 1342 (m), 1279 (m), 1240 (w), 1216 (w), 1146 (m), 1101 (s), 1059 (s), 961 (s), $841(\mathrm{~s}), 668$ (w), $658(\mathrm{w})$.

In the second step, triethylamine $(42 \mu \mathrm{L}, 0.30 \mathrm{mmol})$ was added to a solution of tetra-arm PEG-p-nitrophenylcarbonate as just formed $(0.5 \mathrm{~g}, 0.025 \mathrm{mmol})$ in dimethylformamide $(5 \mathrm{~mL}$, HPLC grade), followed by addition of (S)-(+)-4-(3-aminopyrrolidino)-7-nitrobenzofurazan (NBD) 2 (0.037 g, $0.15 \mathrm{mmol})$. The orange mixture was stirred for $1 \mathrm{~d}$ at room temperature, purified by dialysis in water, and lyophilized to obtain 3 as an orange solid $(0.45 \mathrm{~g}, 85 \%)$. The degree of substitution of the chain-end pendant $p$-nitrophenylcarbonate groups by NBD tags was 
determined to be $92 \%$ by UV absorption spectroscopy; in addition, in ${ }^{1} \mathrm{H}$ NMR, the peaks addressable to $p$-nitrophenylcarbonate have disappeared after this conversion. ${ }^{1} \mathrm{H}$ NMR (400 MHz, DMSO- $\left.d_{6}, \delta\right): 8.24(\mathrm{~d}, J=10.0 \mathrm{~Hz}, 4 \mathrm{H}), 5.75(\mathrm{~d}, J=10.4 \mathrm{~Hz}, 4 \mathrm{H}), 4.55(\mathrm{~s}, 16 \mathrm{H}), 3.53-$ 3.47 (m, 902H, PEG-backbone) ppm.

Scheme S1. Synthesis of four-arm PEG polymers with all chain ends capped by NBD.

(A)

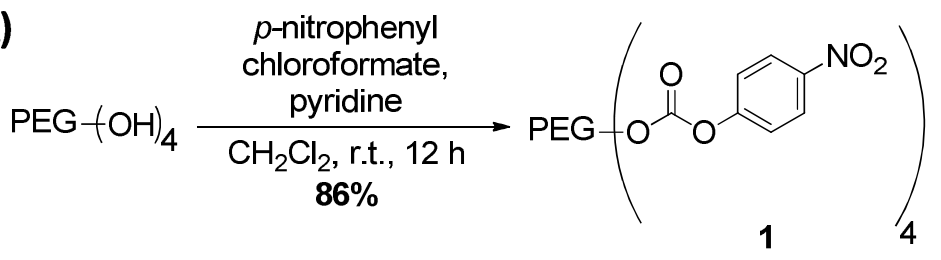

(B)
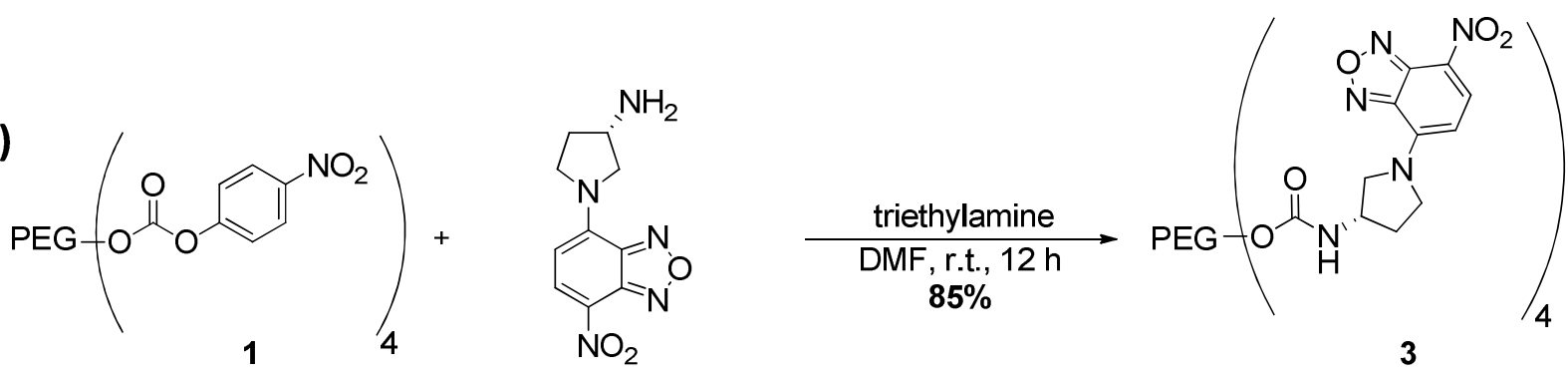

2

\section{B-2. Sample preparation for diffusion measurement}

$1 \mathrm{~mol} \%$ of the NBD-labeled non-associative four-arm PEG was mixed with the gelforming matrix polymers and was dissolved in $500 \mu \mathrm{L}$ of DMF. Separately, $\mathrm{Zn}\left(\mathrm{NO}_{3}\right)_{2} \cdot 6 \mathrm{H}_{2} \mathrm{O}$ in a 2:1 stoichiometric concentration to the PEG polymer was dissolved in $500 \mu \mathrm{L}$ of DMF and vortexed to reach complete dissolution of solids. Equal volumes of the two solutions were then combined in an Eppendorf tube and mixed rigorously with a micro spatula to ensure homogenous mixing. The sample was centrifuged at $21,100 \times \mathrm{g}$ for $10 \mathrm{~min}$ to remove bubbles. 
The sample was sealed between two quartz disks (17 $\mathrm{mm}$ in diameter) separated by a $0.5 \mathrm{~mm}$ thick Teflon spacer. Specimens were equilibrated at $25{ }^{\circ} \mathrm{C}$ for at least 30 min before further experiments were conducted. 


\section{Additional Calculation Details}

\section{C-1. Definition of the volume fraction}

The volume fraction of polymers in the transient network is calculated based on the assumption that the total volume of the gels is the sum of the polymer volume and the solvent volume. In addition, the density of tetra-arm PEG is taken as $1.128 \times 10^{3} \mathrm{~kg} \mathrm{~m}^{-3}$. Therefore, the volume fraction is given by

$$
\varphi=\frac{m_{p} / \rho_{p}}{m_{p} / \rho_{p}+V_{s}}
$$

where the subscripts $p$ and $s$ represent polymer and solvent, respectively, $m$ is the mass, $\rho$ is the density, and $V$ is the volume. In this study, the volume fractions of gels are $0.081,0.117,0.151$, and 0.210 for concentrations $10,15,20$, and $30 \%(\mathrm{w} / \mathrm{v})$, respectively.

\section{C-2. Estimation of the overlap volume fraction of the segment between two stickers $\left(\varphi_{\mathrm{s}}\right)$}

The overlap concentration, $c^{*}$, for the four-arm PEG polymers is $94 \mathrm{~g} \mathrm{~L}^{-1}$, as estimated previously. ${ }^{1}$ Since the stickers are located at the end of the arms in each star polymer, the overlap volume fraction of the segment between two stickers $\left(\varphi_{\mathrm{s}}\right)$ is approximated as the overlap volume fraction of the star-shaped polymers:

$$
\varphi_{\mathrm{s}}=\frac{c^{*}}{\rho}=\frac{1.7 \times 94\left[\mathrm{~g} \mathrm{~L}^{-1}\right]}{1.06 \times 10^{3}\left[\mathrm{~g} \mathrm{~L}^{-1}\right]}=0.089
$$

This calculation suggests that the most of the gel concentrations investigated in this work are above $\varphi_{\mathrm{s}}$ 


\section{C-3. Estimation of the entanglement volume fraction of the segment between two stickers}

$\left(\varphi_{\mathrm{e}}\right)$

Following the classic reptation theory, ${ }^{2}$ the entanglement volume fraction $\varphi_{\mathrm{e}}$ is estimated to be

$$
\varphi_{\mathrm{e}}=\left(\frac{N_{\mathrm{e} 0}}{N}\right)^{3 v-1}
$$

where $N_{\mathrm{e} 0}$ is the number of monomers between entanglements in a melt, $N$ is the degree of polymerization of the polymer, and $v$ is the Flory exponent. The entanglement molecular weight ${ }^{3}$ for PEO is $1,730 \mathrm{~g} \mathrm{~mol}^{-1}$, and the molecular weight of the segment between two stickers is $5,000 \mathrm{~g} \mathrm{~mol}^{-1}$. Therefore,

$$
\varphi_{\mathrm{e}}=\left(\frac{N_{\mathrm{e} 0}}{N}\right)^{3 v-1}=\left(\frac{M_{\mathrm{e} 0}}{M}\right)^{3 v-1}=\left(\frac{1730}{5000}\right)^{3 \times 0.588-1}=0.44
$$

All the gel samples investigated in this study are below $\varphi_{\mathrm{e}}$. This estimation is consistent with the fact that no entanglement plateau is observed in the low-frequency limit in frequency sweep experiments. 


\section{References}

1. Rossow, T.; Habicht, A.; Seiffert, S. Relaxation and Dynamics in Transient Polymer Model Networks. Macromolecules 2014, 47, 6473-6482.

2. Rubinstein, M.; Semenov, A. N. Dynamics of Entangled Solutions of Associating Polymers. Macromolecules 2001, 34, 1058-1068.

3. Fetters, L. J.; Lohse, D. J.; Colby, R. H. Chain Dimensions and Entanglement Spacings. In Physical Properties of Polymers Handbook, Mark, J. E., Ed. Springer New York: New York, NY, 2007; pp 447-454. 